九州大学学術情報リポジトリ

Kyushu University Institutional Repository

\title{
Application of neural networks and fuzzy logic to consumer products
}

Takagi, Hideyuki

Ceut. ral'Research Laboratories, Matsushita Electric Industrial Co., Ltd.

http://hdl. hand le. net/2324/4485347

出版情報: Proceedings of the 1992 International Conference on Industrial Electronics, Control, Instrumentation and Automation. 3, pp. 1629-1633, 1992-11-13. IEEE

バージョン：

権利関係 : 


\title{
APPLICATION OF NEURAL NETWORKS AND FUZZY LOGIC TO CONSUMER PRODUCTS ${ }^{1}$
}

\author{
Hideyuki TAKAGI * \\ Central Research Laboratories, \\ Matsushita Electric Industrial Co., Ltd.
}

\begin{abstract}
This paper describes how neural networks and fuzzy logic have been applied to consumer products. First, the background behind why both technologies have been applied to this field is described. Second, briefly overview of the fusion technology of neural networks and fuzzy logic is given. As a good example of the R\&D process, the application of neural nets to the design and tuning of fuzzy system is introduced. In Sections 4 and 5, applications of both technologies are categorized into four cases. They are: (1) neural networks being used to automate the task of designing and fine-tuning the membership functions of fuzzy systems, (2) both fuzzy inference and neural network learning capabilities provided separately, (3) neural networks work as correcting mechanisms for fuzzy system, (4) neural networks cascaded (serially) with fuzzy sys $1 \mathrm{cms}$, and (5) neural networks used to customize the standard system according to each user's preferences and individual needs. Finally, the new trend that aims at the realization of adaptive systems for the user is discussed. As examples of the trend, four consumer products that apply learning capability of neural networks for the user are introduced.
\end{abstract}

\section{Introduction}

\subsection{Background: Applying Fuzzy Logic}

The use of fuzzy technology has rapidly spread in the realm of consumer product design in order to satisfy the following requirements: (1) to develop control systems with nonlinear characteristics and decision-making systems for controllers, (2) to cope with an increasing number of sensors and exploit the larger quantity of information, (3) to reduce development time, (4) to reduce costs associated with incorporating the technology into the product. Fuzzy technology can satisfy these requirements for the following reasons.

Nonlinear characteristics are realized in fuzzy logic by partitioning the rule space, by weighting the rules, and by the nonlinear membership functions. Rule-based systems compute their output by combining results from different parts of the partition, each part being governed by separate rules. In fuzzy reasoning, the boundaries of these parts overlap, and the local results are combined by wejghting them appropriately. That is why the output of a fuzzy system is a smooth, nonlinear function.

In decision-making systems, the target of modeling is not a control surface but the person whose decision-making is to be emulated. This kind of modeling is outside the realm of conventional control theory. Fuzzy reasoning can tackle this easily since it can

"The author is a Visiting Industrial Fellow at the University of California, Computer Science Division, Berkeley, CA 94720 USA. Tel: +1-510-642-8015, Fax: +1-510-642-5775, e-mail: takagi@cs.berkeley.edu

'Sections 4 and 5 will appear in [1] as the selected paper from the First Int'| Workshop on Industrial Applications of Fuzzy Control and Intelligent Systems, which was held on November 21-22, 1991 in Texas. handle qualitative knowledge (e.g. linguistic terms like 'big' and 'fast', and rules of thumb) directly. In most applications to consumer products, fuzzy systems do not directly control the actuators, but determine the parameters to be used for control. For example, they may determine washing time in washing machines, or if it is is the hand or the image that is shaking in a camcoder, or they compute which object is supposed to be the focus in an autofocus system, or they determine the contrast optimal for watching television.

A fuzzy system encodes knowledge at two levels : knowledge which incorporates fuzzy heuristics, and the knowledge that defines the terms being used in the former level. Due to this separation of meaning, it is possible to directly encode linguistic rules and heuristics. This reduces the development time since the expert's knowledge can be directly built in.

Although the developed fuzzy system may have complex inputoutput characteristics, as long as the mapping is static during the operation of the device, the mapping can be discretized and implemented as a memory lookup on simple hardware. This reduces the costs involved in incorporating the knowledge into the device.

\subsection{Background: Combination with Neural Networks}

One reason for incorporating neural networks into the above process is that the Japanese consumer strongly requires more intelligent and more sensitive appliances with finer capabilities. Manufacturers address this by increasing the number of sensors and the amount of information available to the device. So fuzzy technology is a natural choice for solving this problem.

However, this increase in information leads to a higher complexity of design that cannot be fully tackled by fuzzy reasoning. Neural networks are being introduced to speed up the development of a complex rulebased system. Also, knowledge that cannot be made explicit can be handled by these nets. The first product whose development used neural networks appeared on the market in December 1990. Moreover, there is a further need to have adaptive systems which can be tailored to the user's needs and preferences. Neural networks are expected to be one way to realize this learning capability. This is why both the methodologies of neural nets and fuzzy logic have been combined recently.

\section{Status of Fusion Research of Neural Networks and Fuzzy Logic}

\subsection{Overview}

The earliest research in fusing neural networks and fuzzy logic started in 1974. The main thrust of this research was to use fuzzy logic in neurophysiology. Fuzzy languages, fuzzy entropy, and fuzzy automata were used to model neurons and hearing-vestibular 
nerves and to analyze the nervous system. This has been largely abandoned now.

In the late eighties, especially in 1988, the fusion of neural nets and fuzzy logic began anew. Research has increased dramatically during the nineties. Some of the issues addressed in this research are automatic design and tuning of membership functions, knowledge acquisition and representation, fuzzy cognitive maps, clustering, pattern recognition etc. [2]. From the viewpoint of practical relevance and the quantity of research, the first of these issues is the major one.

\subsection{Design Support of Fuzzy Systems}

In this section, we discuss the developmental path from research in design support to concrete products.

One of the features of fuzzy logic is that it separates the logic from the fuzziness of the terms used. The logic is usually expressed by if-then type of rules, and the fuzziness is captured by defining suitable membership functions for the terms used. Therefore, it is easy to tackle knowledge or skills which can be expressed as rules using qualitative (or linguistic) terms. On the other hand, designing the membership functions (the meaning of the terms) remained difficult and had to be done by trial and error in order to optimize performance. The first research that addressed this difficulty was "NN-driven Fuzzy Reasoning" [3] [4].

First, NN-driven fuzzy reasoning determines the number of rules by clustering the data for designing fuzzy systems. Using this clustered data, a neural network decides on a multidimensional, nonlinear membership function, and this network is then used as a generator of the membership function. One contribution of this research was to introduce neural networks into the design process of fuzzy systems. Secondly, this membership function is designed completely at one stroke, rather than separately along each input axis.

NN-driven fuzzy reasoning can acquire the skill from a human expert automatically. This was demonstrated by the swing-up pole-balancing system designed using this approach [5]. First, a human manually controlled the cart so that the pole was swung up and kept balanced. The demonstration data so collected implicitly included the knowledge of the expert. Extracting this knowledge is difficult from the conventional knowledge engineering standpoint. NN-driven fuzzy reasoning automatically acquired the knowledge, and resulted in a fuzzy system which could duplicate the skill. From a practical viewpoint, this was an important achievement because it reduced the design time of a fuzzy system by using neural networks.

For practical feasibility, the next step is to further reduce the development time. Since designing membership functions is the central theme of the idea and takes the most time, a simplification was proposed to reduce this time [6]. Here, the earlier multidimensional function was decomposed into several one-dimensional functions, which were much easier to design. The error between the designed fuzzy system and the actual data depends on the parameters which define these one-dimensional membership functions. These parameters can then be tuned to minimize the error, in a manner similar to backpropagation learning of weights in a neural network.

The fuzzy system designed as above was applied to the operation of a washing machine, which appeared on the market in early 1991. Following this, similar ideas were used for other kinds of equipment. Details of such techniques and products are presented in Section 4.

\begin{tabular}{|c|c|}
\hline $\begin{array}{l}\text { Matsushita } \\
\text { Electric }\end{array}$ & $\begin{array}{l}\text { air conditioner } \\
\text { washing machine } \\
\text { vacuum cleaner } \\
\text { rice cooker } \\
\text { kerosene fan heater } \\
\text { electric thermo pot } \\
\text { microwave oven } \\
\text { induction heating cooker }\end{array}$ \\
\hline Sanyo & $\begin{array}{l}\text { washing machine } \\
\text { cloth drier } \\
\text { microwave oven } \\
\text { desk type electric heater } \\
\text { electric carpet } \\
\text { electric fan } \\
\text { kerosene fan heater } \\
\text { rice cooker }\end{array}$ \\
\hline Hitachi & $\begin{array}{l}\text { rice cooker } \\
\text { kerosene fan heater } \\
\text { washing machine } \\
\text { vacuum cleaner }\end{array}$ \\
\hline Sharp & $\begin{array}{l}\text { refrigerator } \\
\text { washing machine } \\
\text { rice cooker } \\
\text { kerosene fan heater } \\
\text { forced-flue kerosene fan heater }\end{array}$ \\
\hline Mitsubishi & $\begin{array}{l}\text { kerosene fan heater } \\
\text { induction heating cooker }\end{array}$ \\
\hline Toshiba & $\begin{array}{l}\text { kerosene fan heater } \\
\text { washing machine }\end{array}$ \\
\hline Fujitsu General & kerosene fan heater \\
\hline Corona & kerosene fan heater \\
\hline Toyotomi & kerosene fan heater \\
\hline
\end{tabular}

Table 1: Consumer products in Japan using Neural Networks technology (as of September 20, 1991)

\section{Overview of Applications of Neural Networks and Fuzzy Logic}

In the context of consumer products, neural networks and fuzzy logic have been put to use in the following ways.

\section{Neural Nets as Development Tools for Fuzzy Systems}

\section{Independent Use of Neural Nets and Fuzzy Logic}

3. Neural Nets as Correcting Mechanisms for Fuzzy System

4. Cascade Combination of Neural Nets and Fuzzy System

\section{Learning User Preferences.}

The last way above is a recent trend which uses neural nets to customize the standard system according to each user's preferences and individual needs. The learning capability is being used for adaptation here.

As of September 1991, there were 14 consumer products using neural networks and fuzzy logic in the Japanese market. These are listed in Table 1.

Besides Japan, Korean companies have also been applying these technologies in some of their products. The Korean exhibition [7] displayed an air-conditioner (by Goldstar), washing machines (by Goldstar, Samsun and Daewoo), a kerosene fan heater (by Samsun), and an electric microwave oven range (by Goldstar). 


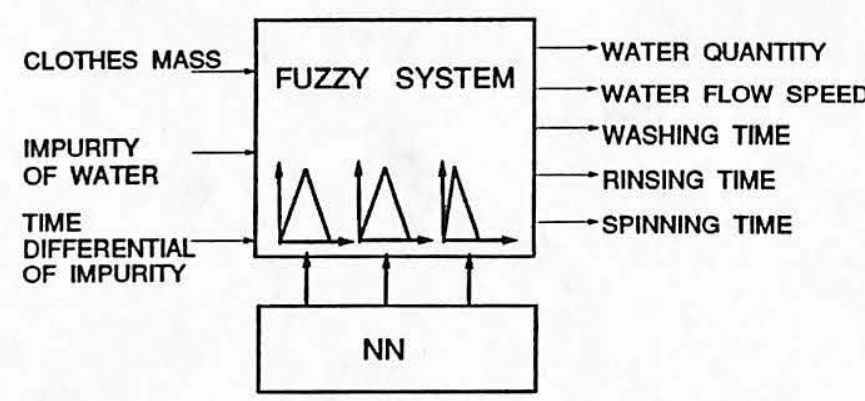

as developing tool

WASHING MACHINE (MATSUSHITA ELECTRIC)

Figure 1: A neural net determines membership functions.

\section{Non-Consumer-Trainable Applications}

\subsection{Development Tools}

Fuzzy logic can encode expert knowledge directly using rules. This uses linguistic (qualitative) labels. However, the precise (quantitative) definitions of these linguistic labels are difficult to obtain, and are often tuned manually, depending on performance. Neural network technology can automate this process of determining the membership function parameters, and can substantially reduce development time while increasing performance. Initial research [3] [4] to this end used partitioning in multi-dimensional spaces. Alternatively, one can use simple-shapes (e.g. triangles) for membership functions, and partition each dimension separately [6]. The latter trades off performance in exchange for faster training.

Consider the inference system in a washing machine, which is shown in Figure 1. It uses three inputs, so a fuzzy partitioning of each of the three one-dimensional input spaces has to be done, and triangular shapes are used to do this. For example, the input variable Clothes-Mass can take the values light, medium and heavy. So three triangles are used to partition the one-dimensional space for this input variable. A similar process is used on the other inputs.

Performance will depend on how this partitioning is done. In other words, the shape and positions of these triangles will affect the output of the system. The partitioning is completely specified by the centers of the triangles and their widths (they are symmetric about their centers). Since performance depends on the parameters of the partitioning, an optimization method is used to determine these so that performance is maximized. Both the fuzzy inference and the optimization process is similar to the way a neural net operates.

Such an approach is being applied to the development of consumer products by the companies of the Matsushita Electric Group.

\subsection{Independent Type}

This approach applies both neural networks and fuzzy logic, but in an unrelated fashion.

One characterization of bodily comfort is that the heat produced by our bodies is absorbed by the surroundings at the same rate, so that we do not accumulate heat or feel cold. The International Standard Organization defines PMV (Predictive Mean Vote) [8] in ISO-7730 as an index of comfort. This is a mathematical function of six input variables, shown on the right side in Figure 4.2. If

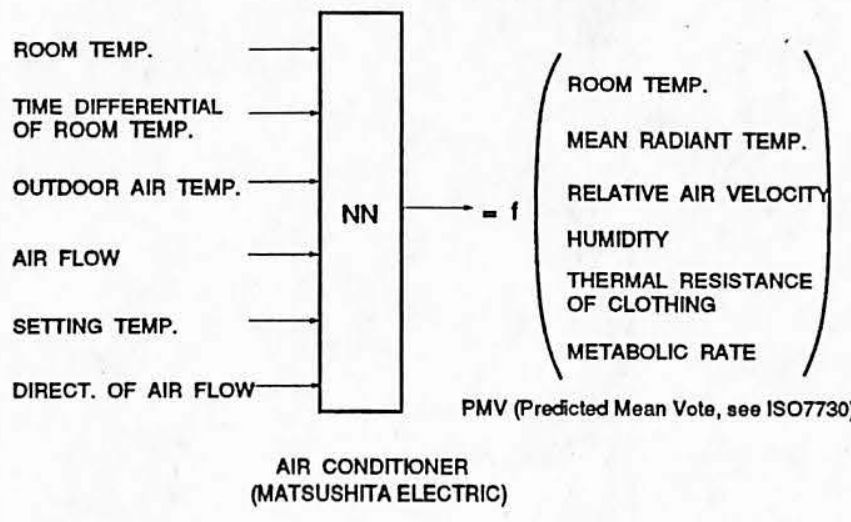

Figure 2: Non-linear mapping from 6-D space to 6-D space

we can compute this function's value, then we can control the airconditioning so as to achieve maximum comfort levels.

However, some of these 6 input variables such as metabolic rate and the thermal resistance of the user's clothing, are difficult to detect by using sensors. Moreover, it would be extremely userunfriendly to ask the user to input these values periodically. In contrast, the variables shown on the left of Figure 4.2 are easy to calculate using sensors.

If we can discover and implement the mapping from the 6 variables on the left to the 6 on the right (a six-dimension to sixdimension mapping), we can estimate the value $f$ of the PMV index using sensor inputs only. This mapping introduces some error since the 6 variables on the left do not completely capture the information provided by the right-hand-side variables. As long as this error in $f$ is within the tolerances allowed for it, we can still use this mapping as a practical method.

The problem now is to discover and calculate this nonlinear relationship between the two sets of variables. Neural nets can handle such a task well, if they are provicled with training data (samples of input-output pairs). The input data is the vector of 6 variables on the left and is sensed. The values of the six on the right are calculated manually (this is the desired output corresponding to the input data). Then the room environment is changed to get another sample (this yields another input-output data pair). Several such samples are used to train the network, which learns the relationship between one set of variables and the other. Now we have a way of finding the value of the PMV index (output of the neural net), and this is used to control the air-conditioner [9]. This is a nice application of the nets' mapping ability, and is being used in the air-conditioners marketed by Matsushita Electric Company.

\section{$4.3 \quad$ Corrector Type}

The application of fuzzy systems technology to Japanese consumer products began in 1990. The next phase involved incorporating more sensor data so as to make the control smoother, sensitive and more accurate. This complicates the task as the input space increases in dimension. In this approach, the neural net handles the larger set of sensor inputs and corrects the output of the fuzzy system (which was designed earlier for the old set of inputs). A complete redesigning of the fuzzy system is thus avoided. This causes a substantial saving in development time (and cost), as redesigning the membership functions becomes more difficult as the number of inputs increases.

Figure 3 shows the schematic underlying the Hitachi washing machine [10]. The fuzzy system shown in the upper part was part of the first model. Later, an improved model incorporated extra information by using a neural net as shown. The neural net pro- 


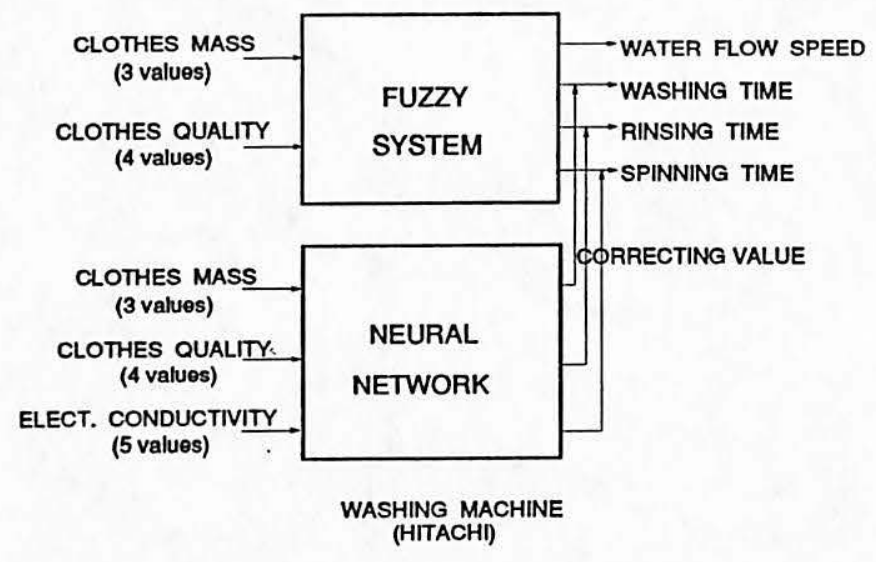

Figure 3: A neural net corrects the output of a fuzzy system

vided corrections to the values output by the fuzzy controller. The additional input (fed only to the net) is Electrical Conductivity, which is used to measure the opacity/transparency of the water. To train the neural net, the desired correction is used. This value is the difference between the desired output and what the fuzzy system outputs. Sanyo uses a similar approach in its washing machine, although some of the inputs/outputs are different [11].

Sanyo has also applied similar methods in the design of its conventional-cum-microwave oven [12]. In the older model, the fuzzy system used the temperature and quantity of the food, and the user-set heater temperature, to compute the heating time, power level to be used, and the boiling point of the food. The newer version eliminates the variation in heating due to changes in the temperature outside the oven, since seasonal changes in temperature do affect the final food temperature slightly. The extra input can be incorporated using a correcting neural network.

\subsection{Cascade Combination Type}

Another way to combine the two technologies is to connect them up serially, where one does a part of the task and then passes its result to the other. In the Sanyo electric fan [13], the fan must rotate towards where the user is, and this requires calculating the direction of the remote controller.

Three infrared sensors in the fan's body detect the strengths of the signal from the remote controller. This information is to be used to compute the direction of the remote control. First, the distance to the remote is calculated by a fuzzy system; then this distance figure and the ratios of sensor outputs are used by a neural network to compute the required direction. The latter calculation is done by a neural net because neither mathematical models nor fuzzy reasoning were successful for doing this computation. The final product has an error of $\pm 4^{\circ}$ as opposed to the $\pm 10^{\circ}$ error of statistical regression methods [14].

\section{Consumer-Trainable Neural Networks}

In the examples given in the previous section, all the neural networks were trained during the design phase. They were then implemented and built into the product, such that the user did not have the option to train them any more. Now we consider products in which the end-user can exploit the trainability of these nets. of the four products described below, information about the last two may be incomplete because their news releases did not reveal detailed technical information regarding the learning capability.

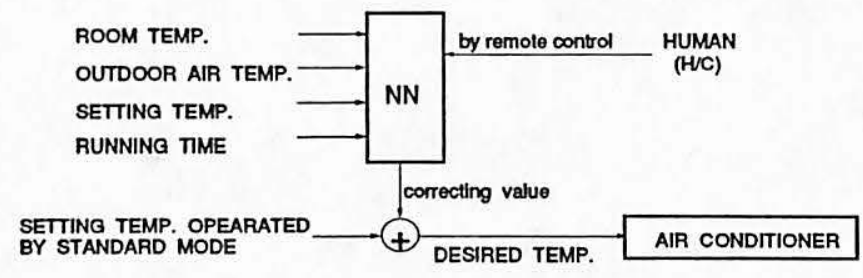

Figure 4: A user-trained network modifies a standard control

\subsection{Reducing Pre-Heating Time}

A product known as a kerosene fan heater is used extensively in Japan to heat the house. The burner burns vaporized oil and this requires pre-heating the burner before it can start heating its surroundings. This pre-heating phase takes considerable time. It would be advantageous if the heater could predict when it was going to be turned on, so that it could start pre-heating accordingly. This prediction depends on the usage pattern which varies from one lome to another. Sanyo uses a neural network to learn this usage pattern [15].

Earlier models of this device used a strong, separate heater ( 600 Watts) to speed up the pre-heating. The model using the neural network to learn the user's pattern took the same time to preheat, but reduced the energy consumption to a half, because of its predictive ability.

The on/off switching of the pre-heating is controlled by a conventional 3-layer feed-forward neural network with 5 hidden units. The three inputs to it are current time (CT), the time elapsed since switching it on $(\Delta T)$, and the previous day's lowest temperature (LT). CT is required to distinguish usage variation between mornings and evenings. $\Delta T$ affects heating power required in the future. Seasonal variation is captured by LT.

Since users can train the network, they may misuse it, causing dangerous accidents. Therefore the manufacturer must pay special attention to consumer safety in the face of misuse. This applies especially to products like the fan heater, which uses combustion. Sanyo has avoided this risk by letting the neural net control only the pre-heating, but not the firing. In the worst case, bad training samples may cause higher energy consumption or inconvenience (longer heating time), but there will be no dangerous consequences of bad training.

This Sanyo heater also uses a fuzzy controller for regulating temperature, and another fuzzy rulebase to estimate the room size.

\subsection{Correction According to Personal Prefer- ence}

The air-conditioner described in Section 4.2 has been augmented by a neural network to further fine-tune the equipment to the user's personal preferences. In the older version, one neural net computed the nonlinear mapping as described before, but this may not be the same as the optimal value for that user. This output is now corrected by a signal produced by the second network, which has been trained by the user. The corrected value is then used in the control of the air-conditioner. The second (user-trained) net uses four inputs from sensors - room temperature, outdoor temperature, temperature setting, and rumning time of the airconditioner. In addition, the user pushes $+/-$ buttons on the remote control to make adjustments for personal comfort. These are an indication of his/her preferences and act as desired output values in supervised training. The output is a correction applied to the output of the first network. See Figure 4 for the schematic diagram. 
Standard learning algorithms such as backpropagation are timeconsuming and may use too much memory, so the above product uses newer and more simplified methods for learning [16]. Current and future trends require the development of new and simpler models to suit the product specifications.

\subsection{Adjusting the Control Program to the User's Environment}

In the case of the fan heater, the characteristics of the space to be heated up, such as size and material (wood or concrete) affect the time needed to heat it up. Ordinarily, the user sets a firing time, keeping in mind the time to warm up. But in the new Sharp heater, the user just has to specify when the room should be warm. The manufacturer cannot set a predesignated time to start pre-heating or firing, since that would depend on the room being warmed up. The factory sets some average standard time only, which is modified by a neural net. Since such equipment (once bought) is generally used in the same room, the neural net learns the heating time by observing it the first few times, and then controls the switching accordingly [17]. So differences in the temperature curve due to room material and size are compensated for.

\subsection{Predicting Pre-Cooling Time}

Another instance of learning usage patterns is found in a refrigerator marketed by Sharp [18]. A higher frequency of door opening/closing of the refrigerator door causes an increase in temperature inside and causes a degradation in the quality of frozen food, which must be kept below $-18^{\circ} \mathrm{C}$. The food still remains frozen, so it is hard to detect the degradation in quality. The refrigerator therefore tries to predict the time when the user increases frequency of door openings, and compensates by pre-cooling the insicles. Both neural nets and fuzzy technology are used to solve this problem.

\section{Requirement for Future Systems}

The introduction of fuzzy and neural teclinology into consumer products from 1990 onwards has led to the introduction of usertrainable networks. The trend is evolving towards more userfriendly and intelligent equipment. The adaptive ability of neural networks makes them invaluable for this purpose.

Having learning functionality in the product is a major improvement, but it alone is not sufficient. Consumer products are targeted towards a wide range of non-specialized users, so incorrect training by them may lead to deterioration in performance, and in the worst case, may be dangerous. Therefore, better safety precautions also need to be built in. One way to handle this requirement is to let the logic ensure strict safety, and let the neural network optimize performance within these safe limits.

One of the realization is to use both implicit and explicit knowledge. It is easy for explicit knowledge to describe rules for the safety of system. The implicit knowledge of neural networks can handle the adaptability for the user. Future advances would involve combining this with high-level cognitive processing, leading to flexible knowledge processing involving fuzzy logic, neural nets, and conventional AI, paving the path to truly intelligent systems.

\section{References}

[1] H. Takagi, "Cooperative system of neural networks and fuzzy logic and its application to consumer products", (ed. J. Yen and R. Langari) Industrial Applications of Fuzzy Control and Intelligent Systems, Van Nostrand Reinhold (will be published in 1993)

[2] H. Takagi, "Fusion technology of fuzzy theory and neural networks - survey and future directions", 1st Int'l Conf. Fuzzy Logic \& Neural Networks (IIZUKA'90), pp.13-26 (July, 1990)

[3] H.Takagi \& I.Hayashi, "Artificial_neural_network-driven fuzzy reasoning", Int'l Workshop on Fuzzy System Applications (IIZUKA'88), pp.217-218 (Aug., 1988)

[4] H.Takagi \& I.Hayashi, "NN-driven fuzzy reasoning", Int'l J. Approximate Reasoning (Special Issue of IIZUKA'88), Vol. 5, No.3, pp.191-212 (1991)

[5] I. Hayashi, H. Nomura and N. Wakami, "Artificial_neural_network-driven fuzzy control and its application to the learning of inverted pendulum system", 3rd IFSA Congress, pp.610-613 (Aug. 1989)

[6] H. Nomura, I. Hayashi, and N. Wakami, "A self-tuning method of fuzzy control by descent method", 4th IFSA Congress, Vol. Engineering, pp.155-158 (1991)

[7] The 1st Joint Conf. \& Exhib. on Artificial Intelligent, Neural Networks, and Fuzzy Systems (Nov. 1991)

[8] P. O. Fanger, "Thermal Comfort - Analysis and Application in Environmental Engineering", McGraw-Hill (1970)

[9] M. Saito, M. Naka, K.Yoshicla, \& I. Akamine, "Estimation of thermal comfort by neural network", Japanese Assoc. of Refrigeration Annual conf., pp.125-128 (1990) (in Japanese)

[10] "Neuro \& Fuzzy logic automatic washing machine and fuzzy logic drier", Hitachi News Release, No.91-024 (Feb. 26, 1991) (in Japanese)

[11] "Neuro \& Fuzzy logic automatic washing machine ASW50v2", Sanyo News Release (Feb. 29, 1991) (in Japanese)

[12] "Two types of Sensor - Oven Range", Sanyo News Release (July 8, 1991) (in Japanese)

[13] "Electric fan series in 1991", Sanyo News Release (Mar., 14, 1991) (in Japanese)

[14] "New trend in consumer electronics: combining neural networks and fuzzy logic", Nikkei Electronics, No.528, pp.165169 (1991.5.25) (in Japanese)

[15] K. Morito, M. Sugimoto, T. Araki, T. Osawa, and Y. Tajima, "Kerosene fan heater using fuzzy control and neural networks 〈CFII-A12JD〉", Sanyo Technical Review, Vol. 23, No. 3, pp.93-100 (1991) (in Japanese)

[16] M. Naka, T. Shida, K. Yoshicla, \& I. Akamine, "Application of neural network for air-conditioner's control", Tech. Report of IEICE, HC91-3T, pp.9-16 (Dec. 1991) (in Japanese)

[17] "Developing 9 type and 19 Kierosene heaters, 1991", Sharp News Release, No.3-032 (June 11, 1991) (in Japanese)

[18] "Slim 66: Marketing a new refrigerator which door opens right/left side freely", Sharp News Release, No.3-053 (Sept. 5,1991 ) (in Japanese) 